\title{
THE DISCONTINUITY POINT SETS OF QUASI-CONTINUOUS FUNCTIONS
}

\section{OLEKSANDR V. MASLYUChENKo}

It is proved that a subset $E$ of a hereditarily normal topological space $X$ is a discontinuity point set of some quasi-continuous function $f: X \rightarrow \mathbb{R}$ if and only if $E$ is a countable union of sets $E_{n}=\bar{A}_{n} \cap \bar{B}_{n}$ where $\bar{A}_{n} \cap B_{n}=A_{n} \cap \bar{B}_{n}=\emptyset$.

\section{INTRODUCTION}

We deal with an old problem of the construction of a function with a given discontinuity point set. Separately continuous functions with given discontinuity point sets were constructed in $[6,5,1,8]$. A complete characterisation of discontinuity point sets for separately continuous functions defined on metrisable spaces was obtained in [9]. An exact problem on construction of functions with given oscillations was considered in $[7,2,4,10,11,12]$. Note that the domain space of all functions was assumed to be metrisable or "near" metrisable in all above papers.

In our note we characterise discontinuity point sets of quasi-continuous functions defined on a hereditarily normal space. We prove in Theorem 4.2 that a subset $E$ of a hereditarily normal topological space $X$ is a discontinuity point set of some quasicontinuous function $f: X \rightarrow \mathbb{R}$ if and only if $E$ is a $\sigma$-junction set (that is, $E$ is a countable union of sets $E_{n}=\bar{A}_{n} \cap \bar{B}_{n}$ where $\bar{A}_{n} \cap B_{n}=A_{n} \cap \bar{B}_{n}=\emptyset$ ).

In [12, Theorem 2.11.1] it was proved that a function $\varphi: X \rightarrow[0,+\infty]$ is the oscillation of some quasi-continuous function $f: X \rightarrow \mathbb{R}$ if and only if the sets $\varphi^{-1}([\varepsilon,+\infty])$ are closed nowhere dense, for each $\varepsilon>0$. But it is easy to see that for any meager $F_{\sigma}$-set $E \subseteq X$ there is a function $\varphi: X \rightarrow[0,+\infty]$ such that $E=\varphi^{-1}(\{0\})$ and the sets $\varphi^{-1}([\varepsilon,+\infty])$ are closed nowhere dense, for each $\varepsilon>0$. So, a subset $E$ of a metrisable space $X$ is a discontinuity point set of some quasi-continuous function $f: X \rightarrow \mathbb{R}$ if and only if $E$ is a meager $F_{\sigma}$-set.

But this fact may to be implied also from our the main result (Theorem 4.2). Indeed, Theorem 2.4 yields that in the metrisable case the $\sigma$-junction set is exactly the meager $F_{\sigma}$-set.

Moreover, in Theorem 4.3 we obtain that if $X$ is a perfectly normal hereditary quasiseparable (or simple, hereditary separable) Frechet-Uryson space then a subset $E$ of $X$

Received 16th October, 2006

Copyright Clearance Centre, Inc. Serial-fee code: 0004-9727/05 \$A2.00+0.00. 
is a discontinuity point set of some quasi-continuous function $f: X \rightarrow \mathbb{R}$ if and only if $E$ is a meager $F_{\sigma}$-set.

\section{JUNCTION SETS}

A subset $E$ of a topological space $X$ we call a junction set if there are subsets $A$ and $B$ of $X$ such that $\bar{A} \cap B=A \cap \bar{B}=\emptyset$ and $E=\bar{A} \cap \bar{B}$.

Proposition 2.1. Let $E$ be a junction set of a topological space $X$. Then $E$ is closed nowhere dense in $X$.

Proof: Let $A$ and $B$ be subsets of $X$ such that $\bar{A} \cap B=A \cap \bar{B}=\emptyset$ and $E=\bar{A} \cap \bar{B}$. Then obviously $E$ is closed. Thus for nowhere density of $E$ it is enough to show that $\operatorname{int} E=\emptyset$. Since int $\bar{A} \cap B \subseteq \bar{A} \cap B=\emptyset$ then $\operatorname{int} \bar{A} \cap \bar{B}=\emptyset$. Hence int $E=\operatorname{int} \bar{A} \cap \operatorname{int} \bar{B}$ $\subseteq \operatorname{int} \bar{A} \cap \bar{B}=\emptyset$.

Under some additional assumptions on $X$ (say, $X$ is metrisable) we shall prove that the junction sets are exactly the closed nowhere dense sets.

Recall some definitions. A family $\left(A_{i}\right)_{i \in I}$ of subsets of a topological space $X$ is said to be discrete (locally finite) if any point of $X$ has a neighbourhood which intersects with at most one (a finite number) of $A_{i}$ 's. A system $\mathcal{A} \subseteq 2^{X}$ is calls $\sigma$-locally finite if $\mathcal{A}$ is a countable union of locally finite systems. A system $\mathcal{A}$ is said to be a net if for any point $x \in X$ and neighbourhood $U$ of $x$ there is $A \in \mathcal{A}$ with $x \in A \subseteq U$. A subset $S \subseteq X$ we call strongly discrete if there exists an discrete family of open sets $U_{s}, s \in S$, such that $s \in U_{s}$ for each $s \in S$. It is easy to see that any closed discrete subset of a paracompact space is strongly discrete. A subset $S \subseteq X$ will be called strongly $\sigma$-discrete if $S$ is a countable union of strongly discrete sets. A subset $E \subseteq X$ we shall call strongly $\bar{\sigma}$-discrete if it contains a dense strongly $\sigma$-discrete subset. A topological space $X$ will be called hereditarily quasi-separable if every subset of $X$ is strongly $\bar{\sigma}$-discrete in $X$.

PROPOSITION 2.2. Let $X$ be a paracompact space with a $\sigma$-locally finite net $\mathcal{A}$. Then $X$ is hereditary quasi-separable.

Proof: Suppose $E \subseteq X$. We set $\mathcal{A}_{E}=\{A \in \mathcal{A}: A \cap E=\emptyset\}$ and for each $A \in \mathcal{A}_{E}$ choose an $s_{A} \in A \cap E$. Since $\mathcal{A}$ is a net then the set $S=\left\{s_{A}: A \in \mathcal{A}_{E}\right\}$ is dense in $E$. By $\sigma$-locally finiteness of $\mathcal{A}$ we obtain that $S$ is a countable union of closed discrete sets. Hence $S$ is strongly $\sigma$-discrete, because $X$ is paracompact. Thus, $E$ is strongly $\bar{\sigma}$-discrete.

Recall that a topological space $X$ is said to be a Frechet-Uryson space if for any $A \subseteq X$ and $x \in \bar{A}$ there exists a sequence of points $x_{n} \in A$ such that $x_{n} \rightarrow x$. An $E \subseteq X$ is called a $\bar{G}_{\delta^{-}}$set if there is a sequence of open sets $G_{n} \supseteq E$ with $E=\bigcap_{n=1}^{\infty} \bar{G}_{n}$

TheOREM 2.3. Let $X$ be a Frechet-Uryson space and $E$ be a closed nowhere dense strongly $\bar{\sigma}$-discrete $\bar{G}_{\delta}$-subset of $X$. Then $E$ is a junction set in $X$. 
Proof: Let $S$ be a dense strongly $\sigma$-discrete subset of $E$ and $S_{n}$ be strongly discrete sets with $S=\bigcup_{n=1}^{\infty} S_{n}$. Without lost of generality we may assume that $S_{n}$ are disjoint. Fix a decreasing sequence of open subsets $G_{n} \supseteq E$ with $E=\bigcap_{n=1}^{\infty} \bar{G}_{n}$. For arbitrary $n \in \mathbb{N}$ pick an open discrete family $(U(s): s \in S)$ so that $s \in U(s) \subseteq \stackrel{n=1}{n}_{n}$ for $s \in S_{n}$. We construct a family $\left(t_{k}(s): k \in \mathbb{N}, s \in S\right)$ so that for arbitrary $k, k^{\prime}, k^{\prime \prime} \in \mathbb{N}$ and $s, s^{\prime}, s^{\prime \prime} \in S$ we have

(1) $t_{k}(s) \in U \backslash E$;

(2) $t_{k^{\prime}}\left(s^{\prime}\right) \neq t_{k^{\prime \prime}}\left(s^{\prime \prime}\right)$ if $\left(k^{\prime}, s^{\prime}\right) \neq\left(k^{\prime \prime}, s^{\prime \prime}\right)$;

(3) $t_{k}(s) \rightarrow s$ as $k \rightarrow \infty$.

Suppose that $t_{k}(s)$ are already constructed for some $m \in \mathbb{N}$ and each $s \in \bigcup_{n<m} S_{n}$ and $k \in \mathbb{N}$ so that (1)-(3) hold. Let us construct $t_{k}(s)$ for $s \in S_{m}$ and $k \in \mathbb{N}^{n<m}$. Put $T_{n}=\left\{t_{k}(s): k \in \mathbb{N}, s \in S_{n}\right\}$ for $n<m$. Since the family $\left(U(s): s \in S_{n}\right)$ is discrete and $S_{n} \cap S_{m}=\emptyset$ for $n<m$ then (1) and (3) imply that $S_{m} \cap \bar{T}_{n}=\emptyset$ for $n<m$. Now fix $s \in S_{m}$. Since $X$ is a Frechet-Uryson space and

$$
s \in \overline{U(s) \backslash\left(E \cup \bigcup_{n<m} T_{n}\right)}
$$

then there is a sequence $t_{k}(s) \in U(s) \backslash\left(E \cup \bigcup_{n<m} T_{n}\right)$ such that $t_{k}(s) \rightarrow s$. Obviously, (1)-(3) are valid.

Now set

$$
\begin{aligned}
A_{n} & =\left\{t_{2 k-1}(s): k \in \mathbb{N}, s \in S_{n}\right\}, \\
B_{n} & =\left\{t_{2 k}(s): k \in \mathbb{N}, s \in S_{n}\right\}, \\
A & =\bigcup_{n=1}^{\infty} A_{n} \text { and } \\
B & =\bigcup_{n=1}^{\infty} B_{n} .
\end{aligned}
$$

Since $\left(U(s): s \in S_{n}\right)$ are discrete then $\bar{A}_{n}=A_{n} \cap S_{n}$ and $\bar{B}_{n}=B_{n} \cap S_{n}$. Taking into account that $U(s) \subseteq G_{n}$ for $s \in S_{n}$ we obtain that $A_{n}, B_{n} \subseteq G_{n}$. Then for each $m \in \mathbb{N}$ one has

$$
\bar{A}=\bigcup_{n>m} \bar{A}_{n} \cup \overline{\bigcup_{n \geqslant m} A_{n}} \subseteq \bigcup_{n<m}\left(A_{n} \cup S_{n}\right) \cup \bar{G}_{m} \subseteq A \cup \bar{G}_{n} .
$$

Recall that $\bigcap_{m=1}^{\infty} \bar{G}_{m}=E$. Hence $\bar{A} \subseteq A \cup E$. Besides,

$$
E=\bar{S}=\overline{\bigcup_{n=1}^{\infty} S_{n}} \subseteq \overline{\bigcup_{n=1}^{\infty} \bar{A}_{n}}=\bar{A} .
$$


Hence $\bar{A}=A \cup E$. Analogously, $\bar{B}=B \cup E$. Thus, $\bar{A} \cap B=A \cap \bar{B}=\emptyset$ and $E=\bar{A} \cap \bar{B}$. Therefore $E$ is a junction set.

By the above results we deduce the following.

THEOREM 2.4. Suppose that for a Frechet-Uryson space $X$ at least, one of the following conditions holds

(i) $X$ is a hereditarily separable perfectly normal;

(ii) $X$ is a hereditarily quasi-separable perfectly normal;

(iii) $X$ is a regular space with a countable net;

(iv) $X$ is a paracompact with a $\sigma$-locally finite net;

(v) $X$ is metrisable.

Then a subset $E$ of $X$ is a junction set in $X$ if and only if $E$ is closed nowhere dense.

Remark that the assumption $X$ be a Frechet-Uryson space is essential in the above two theorems. Indeed, consider the subspace $X=\mathbb{N} \cup\{\varphi\}$ of the Čech-Stone compactification $\beta \mathbb{N}$ of the countable discrete space $\mathbb{N}$ where $\varphi \in \beta \mathbb{N} \backslash \mathbb{N}$. Then $X$ has conditions (i)-(iv) of Theorem 2.4. But nevertheless, $E=\{\varphi\}$ is a closed nowhere dense set in $X$ which is not junction.

\section{A PROOF THAT THE DISCONTINUITY POINT SET OF A QUASI-CONTINUOUS FUNCTION IS A $\sigma$-JUNCTION SET}

We recall that a function $f: X \rightarrow Y$ is called quasi-continuous if $f^{-1}(G)$ $\subseteq \overline{\operatorname{int} f^{-1}(G)}$ for each open $G \subseteq Y$. A subset $E$ of a topological space $X$ we call a $\sigma$-junction set if there is a sequence of junction sets $E_{n}$ with $E=\bigcup_{n=1}^{\infty} E_{n}$.

TheOREM 3.1. Let $X$ be a topological space, $Y$ be a separable metrisable space and $f: X \rightarrow Y$ be a quasi-continuous function. Then the discontinuity point set $D(f)$ of the function $f$ is $\sigma$-junction set.

Proof: Let $\mathcal{V}$ be a countable base of $Y$. Consider a countable system

$$
\{(V, W) \in \mathcal{V} \times \mathcal{V}: \bar{V} \subseteq W\}=\left\{\left(V_{n}, W_{n}\right): n \in \mathbb{N}\right\} .
$$

Set $A_{n}=\operatorname{int} f^{-1}\left(V_{n}\right)$ and $B_{n}=\operatorname{int} f^{-1}\left(Y \backslash \bar{W}_{n}\right)$. Since $f\left(A_{n}\right) \subseteq V_{n}$ and $f\left(B_{n}\right)$ $\subseteq Y \backslash W_{n} \subseteq Y \backslash V_{n}$ then $A_{n} \cap B_{n}=\emptyset$. Taking into account that $A_{n}$ and $B_{n}$ are open one obtains $\bar{A}_{n} \cap B_{n}=A_{n} \cap \bar{B}_{n}=\emptyset$. Thus, $E_{n}=\bar{A}_{n} \cap \bar{B}_{n}$ are junction sets. Besides, $\overline{f\left(A_{n}\right)} \cap \overline{f\left(B_{n}\right)}=\emptyset$ since $\bar{V}_{n} \subseteq W_{n}$. Therefore $E_{n} \subseteq D(f)$. It is left to prove that $D(f) \subseteq \bigcup_{n=1}^{\infty} E_{n}$.

Given $x_{0} \in D(f)$, then there exists a neighbourhood $V$ of $f\left(x_{0}\right)$ with $x_{0}$ $\in \overline{f^{-1}(Y \backslash V)}$. Since $\mathcal{V}$ is a base of $Y$ then there is $n \in \mathbb{N}$ such that $f\left(x_{0}\right) \in V_{n}$ and 
$\bar{W}_{n} \subseteq V$. But $f$ is quasi-continuous. Then $f^{-1}\left(V_{n}\right) \subseteq \bar{A}_{n}$ and

$$
f^{-1}(Y \backslash V) \subseteq f^{-1}\left(Y \backslash W_{n}\right) \subseteq \bar{A}_{n} .
$$

Thus,

$$
x_{0} \in f^{-1}\left(V_{n}\right) \cap \overline{f^{-1}(Y \backslash V)} \subseteq \bar{A}_{n} \cap \bar{B}_{n}=E_{n}
$$

and we obtain $D(f) \subseteq \bigcup_{n=1}^{\infty} E_{n}$.

\section{A CONSTRUCTION OF A QUASI-CONTINUOUS FUNCTION WITH A GIVEN DISCONTINUITY POINT SET}

Recall that a function $f: X \rightarrow \overline{\mathbb{R}}=[-\infty,+\infty]$ is said to be upper (lower) semicontinuous if the set $f^{-1}\left([-\infty, y)\right.$ ) (respectively, $f^{-1}((y,+\infty])$ ) is open for each $y \in \mathbb{R}$. By an upper (lower) Baire function of $f$ we understand the function $f^{*}$ (respectively, $f_{*}$ ) defined by

$$
f^{*}(x)=\inf _{x \in \operatorname{int} U} \sup f(U) \text { and } f_{*}(x)=\sup _{x \in \operatorname{int} U} \inf f(U) \text { for } x \in X .
$$

An upper (lower) Baire function is upper (lower) semi-continuous.

Proposition 4.1. Let $X$ be a topological spaces and $f: X \rightarrow \overline{\mathbb{R}}$ be an lower (upper) semi-continuous function. Then $f^{*}$ (respectively, $f_{*}$ ) is quasi-continuous.

Proof: Let $f$ be, say, lower semi-continuous. Since $f^{*}$ is upper semi-continuous then it remains to prove that $f^{*}$ is lower quasi-continuous (that is, for given $x_{0} \in X$, $y_{0}<f^{*}\left(x_{0}\right)$ and a neighbourhood $U_{0}$ of $x_{0}$ there is an open nonempty set $U \subseteq U_{0}$ such that $f^{*}(x)>y_{0}$ when $\left.x \in U\right)$. Suppose $x_{0} \in X, y_{0}<f^{*}\left(x_{0}\right)$ and let $U_{0}$ be an open neighbourhood of $x_{0}$. Since $y_{0}<f^{*}\left(x_{0}\right) \leqslant \sup f\left(U_{0}\right)$ then there is an $x_{1} \in U_{0}$ with $f\left(x_{1}\right)>y_{0}$. But $f$ is lower semi-continuous. Then there exists an open neighbourhood $U \subseteq U_{0}$ of $x_{1}$ such that $f(x)>y_{0}$ on $U$. Finally, $f^{*}(x) \geqslant f(x)>y_{0}$ on $U$.

TheOREM 4.2. Let $X$ be a hereditarily normal space and $E \subseteq X$. Then $E$ is a discontinuity point set of some quasi-continuous function $f: X \rightarrow \mathbb{R}$ if and only if $E$ is a $\sigma$-junction set.

Proof: Let $E_{n}$ be junction sets such that $E=\bigcup_{n=1}^{\infty} E_{n}$. Choose sets $A_{n}$ and $B_{n}$ such that $\bar{A}_{n} \cap B_{n}=A_{n} \cap \bar{B}_{n}=\emptyset$ and $E_{n}=\bar{A}_{n} \cap \bar{B}_{n}$. Fix $n \in \mathbb{N}$. Since $X \backslash E_{n}$ is normal, $A_{n} \cap E_{n}=B_{n} \cap E_{n}=\emptyset$ and $\left(\bar{A}_{n} \cap \bar{B}_{n}\right) \backslash E_{n}=\emptyset$ then by the Uryson lemma [3] there exists a continuous function $\tilde{f}_{n}: X \backslash E_{n} \rightarrow[0,1]$ such that $\tilde{f}_{n}(x)=0$ on $A_{n}$ and $\tilde{f}_{n}(x)=1$ on $B_{n}$. Define $f_{n}(x)=0$ for $x \in E_{n}$ and $f_{n}(x)=\tilde{f}_{n}(x)$ for $x \in X \backslash E_{n}$. Since $\overline{f\left(A_{n}\right)} \cap \overline{f\left(B_{n}\right)}=\emptyset$ and $E_{n}=\bar{A}_{n} \cap \bar{B}_{n}$ then $E_{n} \subseteq D\left(f_{n}\right)$. But $\left.f\right|_{X \backslash E_{n}}$ is continuous. Thus $E_{n}=D\left(f_{n}\right)$. Besides, the points $x \in E_{n}=D\left(f_{n}\right)$ are the minimum points of $f_{n}$. Therefore $f_{n}$ is lower semi-continuous. 
Define $g=\sum_{n=1}^{\infty}\left(1 / 4^{n}\right) f_{n}$ and $f=g^{*}$. Since $g$ is lower semi-continuous then $f$ is quasicontinuous by Proposition 4.1. Let us prove that $D(f)=E$. First, if all $f_{n}$ are continuous at a given point $x_{0} \in X$ then so is $g$, and hence $f$. Thus, $E=\bigcup_{n=1}^{\infty} D\left(f_{n}\right) \supseteq D(f)$.

Given $x_{0} \in E$, we show that $x_{0} \in D(f)$. Let $n$ be the least integer such that $x_{0} \in E_{n}$. We set $\varepsilon=1 / 4^{n}, u=\sum_{k<n}\left(1 / 4^{k}\right) f_{k}$ and $v=\sum_{k>n}\left(1 / 4^{k}\right) f_{k}$. Since $x_{0} \notin E_{k}=D\left(f_{k}\right)$ for $k<n$ then $u$ is continuous at $x_{0}$. Therefore there is an open neighbourhood $U$ of $x_{0}$ such that $\left|u(x)-u\left(x_{0}\right)\right|<\varepsilon / 9$ on for $x \in U$. Put $G=U \cap \operatorname{int} f_{n}^{-1}([0,1 / 9))$ and $H=U \cap \operatorname{int} f_{n}^{-1}((8 / 9,1])$. Since $f_{n}$ is continuous on $X \backslash E_{n} \supseteq A_{n}, B_{n}$ and $f_{n}(x)=0$ on $A_{n}$ and $f_{n}(x)=1$ on $B_{n}$ then $A_{n} \cap U \subseteq G$ and $B_{n} \cap U \subseteq H$. Hence $x_{0} \in \bar{G} \cap \bar{H}$. Besides,

$$
u(x) \leqslant \sum_{k>n} \frac{1}{4^{k}}=\frac{1 / 4^{n+1}}{1-(1 / 4)}=\frac{1}{3 \cdot 4^{n}}=\frac{\varepsilon}{3}
$$

for $x \in X$. Thus for $x \in G$ one has

$$
g(x)=u(x)+\varepsilon f_{n}(x)+v(x)<u\left(x_{0}\right)+\frac{\varepsilon}{9}+\frac{\varepsilon}{9}+\frac{\varepsilon}{3}=u\left(x_{0}\right)+\frac{5 \varepsilon}{9} .
$$

For $y \in H$ we obtain the converse inequality

$$
g(y) \geqslant u(y)+\varepsilon f_{n}(y)>u\left(x_{0}\right)-\frac{\varepsilon}{9}+\frac{8 \varepsilon}{9}=u\left(x_{0}\right)+\frac{7 \varepsilon}{9} .
$$

But since $f=g^{*}$ and the sets $G$ and $H$ are open then for any $x \in G$ and $y \in H$

$$
f(x) \leqslant u\left(x_{0}\right)+\frac{5 \varepsilon}{9}<u\left(x_{0}\right)+\frac{7 \varepsilon}{9} \leqslant f(y) .
$$

From $x_{0} \in \bar{G} \cap \bar{H}$ we finally obtain that $f$ is discontinuous at $x_{0}$.

Theorems 2.4 and 4.2 together imply the following.

THEOREM 4.3. Let for a Frechet-Uryson space $X$ at least, one of the following conditions holds

(i) $X$ is a hereditarily separable perfectly normal;

(ii) $X$ is a hereditarily quasi-separable perfectly normal;

(iii) $X$ is a regular space with a countable net;

(iv) $X$ is a paracompact with a $\sigma$-locally finite net;

(v) $X$ is metrisable.

Then a subset $E$ of $X$ is the discontinuity point sets of some quasi-continuous function $f: X \rightarrow \mathbb{R}$ if and only if $E$ is a meager $F_{\sigma}$-set. 


\section{REFERENCES}

[1] J.C. Breckenridge and T. Nishiura, 'Partial continuity, quasi-continuity and Baire spaces', Bull. Inst. Math. Acad. Sinica. 4 (1976), 191-203.

[2] Z. Duszynski, Z. Grande, and S. Ponomarev, 'On the w-primitives', Math. Slovaca 51 (2001), 469-476.

[3] R. Engelking, General topology, (Russian) (Mir, Moscow, 1986).

[4] J. Ewert and S. Ponomarev, 'Oscillation and w-primitives', Real Anal. Exchange 26 (2001), 687-702.

[5] Z. Grande, 'Une caractérisation des ensembles des point de discontinuité des fonctions linéairement-continues', Proc. Amer. Math. Soc. 52 (1975), 257-262.

[6] R. Kershner, 'The continuity of functions of many vatiables', Trans. Amer. Math. Soc. 53 (1943), 83-100.

[7] P. Kostyrko, 'Some properties of oscillation', Math. Slovaca. 30 (1980), 157-162.

[8] V.K. Maslyuchenko and V.V. Mykhaylyuk, 'On separately continuous functions defined on products of metrizable spaces', (Ukrainian), Dopovidi Akad. Nauk. Ukrajini (1993), 28-31.

[9] V.K. Maslyuchenko and V.V. Mykhaylyuk, 'Charecterization of sets of discontinuity point of separately continuous functions of several variables on products of metrizable spaces', (Ukrainian), Ukrainian Math. Zh. 52 (2000), 740-747.

[10] V.K. Maslyuchenko and O.V. Maslyuchenko, 'Construction of a separately continuous function a with given oscillation', (Ukrainian), Ukrainian Math. J. 50 (1998), 948-959.

[11] V.K. Maslyuchenko, O.V. Maslyuchenko, V.V. Mykhaylyuk and O.V. Sobchuk, 'Paracompactness and separately continuous mappings', in General Topology in Banach Spañes, (T. Banakh and A. Plichko, Editors) (Nova Sci. Publ., Huntington, New York, 2001), pp. 147-169.

[12] O.V. Maslyuchenko, The oscillation of separately continuous functions and topological games (Ukrainian), (Dissertation) (Chernivtsi, 2002).

Department of Mathematic

Jurij Fed'kovych Chernivtsi National University

vul Kotsyubyns'kolio 2

Cherniutsi 58012

Ukraine

e-mail: mathan@chnu.cv.ua 